\title{
Retraction Note to: MiR-154 Functions as a Tumor Suppressor in Glioblastoma by Targeting Wnt5a
}

\author{
Dongsheng Zhao ${ }^{1} \cdot$ Rencong Wang ${ }^{1} \cdot \mathrm{Junkang} \mathrm{Fang}^{2} \cdot$ Xituan $\mathrm{Ji}^{1} \cdot \mathrm{Juan} \mathrm{Li}^{1}$ • \\ Xiaoyan Chen ${ }^{1}$ - Gangfeng Sun ${ }^{1} \cdot$ Zhengjun Wang $^{1}$ • Weiping Liu ${ }^{1}$ - Yangang Wang ${ }^{1}$. \\ Guang Cheng ${ }^{1} \cdot$ Haining Zhen ${ }^{1} \cdot$ Chunhua Sun ${ }^{3} \cdot$ Zhou Fei $^{1}$
}

Published online: 5 July 2017

(C) Springer Science+Business Media, LLC 2017

Retraction Note to: Mol Neurobiol (2017) 54:2823-2830

DOI 10.1007/s12035-016-9867-5

This article has been retracted at the request of the Editor-inChief and the Publisher per the Committee on Publication Ethics guidelines. The article shows evidence of irregularities in authorship during the submission process, there is strong reason to believe that the peer review process was compromised and the authors have plagiarized parts from the following article:

Xinsheng Liu, Zhiyong Liang, Kehai Gao, Huazhuang Li,

Guangzong Zhao, Shantao Wang, Jun Fang, MicroRNA-

128 inhibits EMT of human osteosarcoma cells by direct-

ly targeting integrin $\alpha 2$, Tumor Biology, June 2016,

Volume 37, Issue 6, pp 7951-7957, DOI: 10.1007/

s13277-015-4696-0; Received: 28 November 2015

As such the validity of the content of this article cannot be verified.

The online version of the original article can be found at http://dx.doi.org/ 10.1007/s12035-016-9867-5

Chunhua Sun

actively0126@126.com

Zhou Fei

feizhou@fmmu.edu.cn

1 Department of Neurosurgery, Xijing Hospital, Fourth Military Medical University, Xi'an, Shaanxi 710032, China

2 Department of Neurosurgery, Dongyang People's Hospital of Zhejiang Province, Dongyang, Zhejiang 322100, China

3 Department of Oncology, Nanjing Medical University Affiliated Wuxi Second Hospital, Wuxi 214002, China 